\title{
Pulmonary hypertension in advanced sarcoidosis: epidemiology and clinical characteristics
}

\author{
A.F. Shorr*, D.L. Helman*, D.B. Davies" ${ }^{\#}$ and S.D. Nathan
}

ABSTRACT: Pulmonary hypertension $(\mathrm{PH})$ is a predictor of poor outcome in sarcoidosis. Little is known about the epidemiology of $\mathrm{PH}$ in sarcoidosis.

The current authors reviewed the records of patients with sarcoidosis listed for lung transplantation in the USA between January 1995 and December 2002. PH was defined as a mean pulmonary artery pressure of $>25 \mathrm{mmHg}$ and severe $\mathrm{PH}$ as a mean pulmonary artery pressure of $\geqslant \mathbf{4 0} \mathrm{mmHg}$.

The cohort included 363 patients of whom $73.8 \%$ had $\mathrm{PH}$. Neither spirometric testing nor the need for corticosteroids was associated with $\mathrm{PH}$. Subjects with $\mathrm{PH}$ required more supplemental oxygen $\left(2.7 \pm 1.8 \mathrm{~L} \cdot \mathrm{min}^{-1}\right.$ versus $\left.1.6 \pm 1.4 \mathrm{~L} \cdot \mathrm{min}^{-1}\right)$. The cardiac index was lower in individuals with $\mathrm{PH}$, whereas the pulmonary capillary wedge pressure was higher. In multivariate analysis, supplemental oxygen remained an independent predictor of $\mathrm{PH}$, whereas the relationship between cardiac index and PH was no longer significant. As a screening test, the need for oxygen had a sensitivity and specificity of $91.8 \%$ and $32.6 \%$, respectively.

Pulmonary hypertension is common in advanced sarcoidosis. The need for oxygen correlates with pulmonary hypertension. Since pulmonary hypertension is associated with poor outcomes and because simple clinical criteria fail to identify patients with sarcoidosis and pulmonary hypertension, more aggressive screening for this should be considered.

KEYWORDS: Epidemiology, oxygen, pulmonary hypertension, sarcoidosis, transplantation

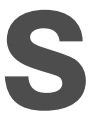
arcoidosis is a disease of unknown aetiology, which has protein manifestations. Although any organ system may be involved, sarcoidosis almost uniformly affects the lungs and intrathoracic lymph nodes. The majority of patients with sarcoidosis do well, with few requiring extended therapy $[1,2]$. For those who do need regular treatment, the list of available alternatives continues to expand and now includes methotrexate and infliximab, in addition to corticosteroids [3-5]. Despite these recent advances, some individuals do poorly and develop end-stage lung disease. In such cases, orthotopic lung transplantation (OLT) has emerged as an important consideration.

The decision to list a patient for OLT incorporates an estimate of their expected intermediate-term survival, e.g. over the next 2 yrs [5]. As with many forms of interstitial lung disease, predicting survival is difficult given both the variable natural history of the process and the limited published experience with risk stratification [6]. However, several recent reports have documented poor outcomes among patients with sarcoidosis who develop pulmonary hypertension $(\mathrm{PH})[7,8]$. ARCASOY et al. [7], in a review of all sarcoidosis patients at their institution listed for OLT, found that survival was significantly worse in those who had right atrial pressures $>15 \mathrm{mmHg}$. In a similar analysis, the current authors noted that the presence of $\mathrm{PH}$ adversely affected survival [8]. Proposed changes to the organ allocation system in the USA for lung transplantation have incorporated these findings [9]. These recommendations give added priority to patients with sarcoidosis who suffer from $\mathrm{PH}$.

Due to the importance of $\mathrm{PH}$ as a determinant of prognosis in sarcoidosis, coupled with the potential priority given to these subjects in terms of undergoing OLT, it was hypothesised that
AFFILIATIONS

*The Pulmonary and Critical Care Medicine Service, Walter Reed Army Medical Center, Washington, DC, \#The United Network for Organ Sharing, Richmond, and

"The Inova Fairfax Transplant Center Falls Church, VA, USA.

CORRESPONDENCE

A.F. Shorr

9005 Cherbourg Dr.

Potomac

MD 20854

USA

Fax: 12027829032

E-mail: afshorr@dnamail.com

Received

July 122004

Accepted after revision:

December 142004

\section{SUPPORT STATEMENT}

The opinions expressed herein are not to be construed as official or as reflecting the policies of either the Dept of Defense or the Dept of the Army 
readily available clinical characteristics would identify patients with sarcoidosis likely to concomitantly have elevated pulmonary arterial pressures $(P$ pa). A retrospective analysis of all patients listed for OLT with sarcoidosis in the USA was conducted in order to determine which factors were associated with the presence of $\mathrm{PH}$ and to assess the accuracy of these variables in identifying patients with sarcoidosis and $\mathrm{PH}$.

\section{METHODS}

\section{Subjects and end-points}

For all subjects listed for OLT in the USA, the United Network for Organ Sharing (UNOS) oversees the listing process for organ allocation. As part of this responsibility, UNOS maintains a registry of all patients eventually listed for OLT and their clinical characteristics. The current authors reviewed this registry and identified patients listed for OLT irrespective of type of proposed transplant (single lung, double lung and heart-lung) for sarcoidosis during the 7 yrs between January 1995 and December 2002. Patients <18 yrs of age were excluded and the diagnosis of sarcoidosis was based on the reports of the referring transplant centre. The results of invasive right heart catheterisation (RHC) were utilised to categorise patients as to the presence or absence of $\mathrm{PH}$. The current authors defined $\mathrm{PH}$, the primary end-point, as the presence of mean Ppa $>25 \mathrm{mmHg}$. Severe PH (e.g. mean Ppa of $\geqslant 40 \mathrm{mmHg}$ ) represented a secondary end-point. Initial demographic and outcomes data from segments of this cohort have been reported elsewhere $[8,10]$.

\section{Study variables}

In order to list a patient for OLT with UNOS, the referral centre provides a comprehensive profile of the subject, describing the demographic features, pulmonary function, functional status and comorbid medical illnesses. For the present analysis, specific variables of interest included patient age, sex and race. For pulmonary function and physiology, the relationship between $P$ pa and forced vital capacity (FVC), forced expiratory volume in one second (FEV1), carbon dioxide arterial tension $\left(\mathrm{Pa}, \mathrm{CO}_{2}\right)$, need for supplemental oxygen, and 6-min walk distance were explored. UNOS does not record data on the arterial oxygen tension $\left(\mathrm{Pa}, \mathrm{O}_{2}\right)$ and the diffusion capacity of carbon monoxide $(\mathrm{DL}, \mathrm{CO})$. Additionally, UNOS did not begin to require reporting of the FEV1/FVC ratio until 1999. Therefore, this factor was not included in the present assessment. From the invasive testing, the authors collected measures of cardiac performance (e.g. cardiac index) and the pulmonary capillary wedge pressure $(P \mathrm{pcw})$.

With respect to functional status, patients were categorised as needing no assistance, some assistance, or complete assistance with their activities of daily living. The patient's employment status (working versus unemployed), location at time of listing (e.g. outpatient versus hospitalised) and whether they required life support, such as vasoactive medications or mechanical ventilation, were all determined. Although UNOS offers no specific guidance for the management of sarcoidosis, referral centres may classify patients as being corticosteroid dependent and, thus, this factor was also examined. For comorbid illnesses, the current authors focused on the presence or absence of vascular diseases, such as hypertension, angina and stroke.

\section{Statistics}

Univariate analyses were performed for all the variables noted above, comparing those with $\mathrm{PH}$ to those with normal $P$ pa and then comparing patients with severe $\mathrm{PH}$ to those with $P$ pa $\leqslant 25 \mathrm{mmHg}$. Continuous data are reported as mean \pm SD. Categorical variables were compared with the Chi-squared test. The present study evaluated continuous variables with an unpaired t-test if normally distributed or with a Wilcoxon rank sum test if they followed a non-normal distribution. All tests were two-tailed and a p-value of $<0.05$ was assumed to represent statistical significance.

All variables were initially placed in a multivariate logistic regression model to identify factors associated with $\mathrm{PH}$ and severe PH. As a sensitivity analysis and to explore this approach to multivariate analysis, a second model was created. In the second model, variables that were significant at the $\mathrm{p}=0.2$ level in the univariate analysis were included as covariates in a backward elimination, multivariable logistic regression in order to determine which factors were independently associated with $\mathrm{PH}$ and severe $\mathrm{PH}$. The results of the second model were not different from the initial analysis; hence, only the results of the model with all variables entered are reported herein. To determine the implications of these predictors of $\mathrm{PH}$, which emerged from the logistic regression, the authors computed their sensitivity, specificity, positive predictive value and negative predictive value as a function of their ability to identify patients with $\mathrm{PH}$. Where appropriate, 95\% confidence intervals (CI) were reported.

\section{RESULTS}

During the study period, there were 363 patients with sarcoidosis listed for OLT and who had complete RHC data available. This represents $72.5 \%$ of all sarcoidosis subjects listed, and those without results from RHC did not differ from those undergoing RHC with respect to any of the clinical variables analysed (data not shown). The mean age of the cohort was 46 yrs, $65 \%$ were female and nearly $70 \%$ of patients were African American. PH was common and noted in $73.8 \%$ of the population. Severe $\mathrm{PH}$ was reported in more than a third $(n=131,36 \%)$ of cases.

As shown in table 1, demographic variables were not related to the presence of $\mathrm{PH}$. For example, $\sim 70 \%$ of Caucasians had elevated $P$ pa as compared with $76.2 \%$ of African Americans. Results of pulmonary function tests also failed to differ based on the presence or absence of $\mathrm{PH}$. However, patients with $P$ pa $>25 \mathrm{mmHg}$ required, on average, more supplemental oxygen. The mean $\mathrm{O}_{2}$ requirement in those with $\mathrm{PH}$ was $2.7 \pm 1.8 \mathrm{~L} \cdot \mathrm{min}^{-1}$ versus $1.6 \pm 1.4 \mathrm{~L} \cdot \mathrm{min}^{-1} \quad(\mathrm{p}<0.001)$. Additionally, functional status and employment status varied in relation to $P$ pa. Subjects with normal pulmonary pressures were 1.74-times (95\% CI: 1.07-2.83) more likely to report no limitations with functional status than patients with $P_{p a}$ $>25 \mathrm{mmHg}$. Conversely, $\mathrm{PH}$ doubled the risk that an individual with sarcoidosis would be unemployed due to disease (odds ratio (OR): 2.08; 95\% CI: 1.30-3.42). Hospitalisation at time of listing and the need for life support (e.g. therapy with vasopressor and/or mechanical ventilation) were infrequent and failed to correlate with the results of RHC. Systemic hypertension necessitating treatment was more common among patients with $P$ pa $>25 \mathrm{mmHg}$. Nearly $25 \%$ 
TABLE 1 Correlates of pulmonary hypertension

\begin{tabular}{|c|c|c|c|c|c|}
\hline Variable & $P p a \leqslant 25 \mathrm{mmHg}$ & $P$ pa $>25 \mathrm{mmHg}$ & $P p a \geqslant 40 \mathrm{mmHg}$ & $\begin{array}{c}\text { p-value } \\
(P \text { pa }>25 \mathrm{mmHg} \text { versus } \\
\leqslant 25 \mathrm{mmHg})\end{array}$ & $\begin{array}{c}\text { p-value } \\
(P \text { pa } \geqslant \mathbf{4 0 ~} \mathrm{mmHg} \text { versus } \\
\leqslant 25 \mathrm{mmHg})\end{array}$ \\
\hline Subjects n & 95 & 268 & 131 & & \\
\hline \multicolumn{6}{|l|}{ Demographics } \\
\hline Age yrs & $45.6 \pm 8.8$ & $46.5 \pm 7.8$ & $46.0 \pm 7.2$ & 0.323 & 0.684 \\
\hline \multicolumn{6}{|l|}{ Pulmonary function } \\
\hline FVC \% pred & $44.7 \pm 15.2$ & $46.4 \pm 16.2$ & $49.8 \pm 17.3$ & 0.373 & 0.024 \\
\hline FEV $1 \%$ pred & $38.7 \pm 14.9$ & $39.5 \pm 16.1$ & $43.7 \pm 17.1$ & 0.651 & 0.025 \\
\hline $\mathrm{Pa}, \mathrm{CO}_{2} \mathrm{mmHg}$ & $41.3 \pm 7.3$ & $43.1 \pm 9.4$ & $40.9 \pm 9.0$ & 0.108 & 0.715 \\
\hline 6-min walk distance $<52.2 \mathrm{~m} \%$ & 9.5 & 13.1 & 12.2 & 0.357 & 0.517 \\
\hline Any need for $\mathrm{O}_{2} \%$ & 67.3 & 91.8 & 93.4 & $<0.001$ & $<0.001$ \\
\hline Working \% & 78.6 & 76.5 & 61.1 & 0.004 & 0.004 \\
\hline Some or total ADL assistance \% & 52.6 & 62.7 & 68.7 & 0.086 & 0.014 \\
\hline Hospitalised \% & 2.1 & 3.4 & 6.1 & 0.540 & 0.662 \\
\hline $\ln I C U \%$ & 2.1 & 3.4 & 4.6 & 0.540 & 0.320 \\
\hline \multicolumn{6}{|l|}{ Comorbid illnesses } \\
\hline Hypertension \% & 3.2 & 25.7 & 29.8 & 0.016 & 0.005 \\
\hline Angina \% & 3.2 & 4.1 & 2.3 & 0.681 & 0.689 \\
\hline Stroke \% & 2.1 & 0.4 & 0.8 & 0.169 & 0.574 \\
\hline Corticosteroids ${ }^{\#} \%$ & 53.7 & 59.7 & 55.0 & 0.307 & 0.849 \\
\hline \multicolumn{6}{|l|}{ Haemodynamic monitoring } \\
\hline Cardiac index $\mathrm{L} \cdot \mathrm{min}^{-1} \cdot \mathrm{m}^{-2}$ & $2.79 \pm 0.54$ & $2.66 \pm 3.58$ & $2.38 \pm 0.74$ & 0.030 & 0.009 \\
\hline Ppcw mmHg & $7.78 \pm 0.79$ & $12.69 \pm 7.51$ & $14.18 \pm 8.94$ & 0.007 & 0.005 \\
\hline
\end{tabular}

of those with $\mathrm{PH}$ were being treated for systemic hypertension, whereas only $13.7 \%$ of those lacking $\mathrm{PH}$ were receiving therapy for an elevated blood pressure $(p=0.016)$.

The cardiac function was statistically lower in those with $\mathrm{PH}$ with the cardiac index measuring $2.66 \pm 3.58 \mathrm{~L} \cdot \mathrm{min}^{-1} \cdot \mathrm{m}^{-2}$ whereas it was $2.79 \pm 0.54 \mathrm{~L} \cdot \mathrm{min}^{-1} \cdot \mathrm{m}^{-2}$ in patients with normal $P_{\mathrm{pa}}(\mathrm{p}=0.03)$. The $P_{\mathrm{pcw}}$ was concomitantly higher in the setting of $\mathrm{PH}(12.69 \pm 7.51 \mathrm{mmHg}$ versus $7.78 \pm 0.79 \mathrm{mmHg}, \mathrm{p}=0.009)$.

Table 1 also reveals the results of the comparisons of those with severe $\mathrm{PH}$ to subjects with normal $P$ pa. Variables associated with $P$ pa $>25 \mathrm{mmHg}$ correlated with the presence of severe $P$ pa elevations. In addition, restricting the analysis to this cohort demonstrates a univariate association between pulmonary function tests and $P$ pa. The FVC was higher whereas the $P \mathrm{a}, \mathrm{CO}_{2}$ was lower in patients with severe $\mathrm{PH}$. Differences in cardiac index and $\mathrm{Ppcw}$ became more pronounced when the analysis was restricted to patients with severe $\mathrm{PH}$.

In multivariate analysis, only two variables were associated with the presence of $\mathrm{PH}$. For each $\mathrm{L} \cdot \mathrm{min}^{-1}$ increase in needed oxygen flow, the risk of $\mathrm{PH}$ approximately doubled (OR: 2.47; 95\% CI: 1.61-3.79). When analysed as a categorical variable in separate models, both need for any supplemental oxygen and use of $\geqslant 3 \mathrm{~L} \cdot \mathrm{min}^{-1}$ were independently related to the presence of $\mathrm{PH}$ (OR: 8.39, 95\% CI: 3.44-20.47 for any $\mathrm{O}_{2}$; OR: 3.04, 95\% CI: $1.47-6.31$ for $\mathrm{O}_{2} \geqslant 3 \mathrm{~L} \cdot \mathrm{min}^{-1}$ ). Ppcw also independently correlated with $\mathrm{PH}$. For each $1-\mathrm{mmHg}$ increase in Ppcw above the mean value for the cohort, the likelihood of $\mathrm{PH}$ increased by $29 \%$ (OR: $1.29 ; 95 \%$ CI: $1.18-1.41)$.

For severe $\mathrm{PH}$, the independent probability of having this condition also doubled with each $\mathrm{L} \cdot \mathrm{min}^{-1}$ increase in the use of oxygen (OR: 2.56; 95\% CI: 1.03-6.24). Patients with severe $\mathrm{PH}$ were nearly seven times more likely (OR: 10.28; 95\% CI: $2.67-$ $39.24)$ to require supplemental oxygen. Need for treatment for systemic hypertension negatively correlated with the presence of severe PH (OR: 0.28; 95\% CI: 0.08-0.94). The cardiac index was more impaired in the case of severe $\mathrm{PH}$. With each $1-\mathrm{L} \cdot \mathrm{min}^{-1} \cdot \mathrm{m}^{-2}$ increase in this, compared to the mean cardiac index for the cohort, the probability of severe $\mathrm{PH}$ decreased by $34 \%$ (95\% CI: 0.17-0.70). 
Although oxygen need was independently associated with $\mathrm{PH}$, its predictive power was limited. For example, employing the need for supplemental oxygen as a screening test to identify subjects suffering from $P$ pa $>25 \mathrm{mmHg}$ had a sensitivity and specificity of 91.8 and $32.6 \%$, respectively. The positive and negative predictive values measured 79.4 and $58.5 \%$, respectively. Thus, the accuracy (true positive+true negative rate) equalled only $68.6 \%$. Increasing the threshold to use of $\geqslant 3 \mathrm{~L} \cdot \mathrm{min}^{-1}$ compromised sensitivity while improving specificity (44.9 and $77.9 \%$, respectively). The overall accuracy decreased to $52.9 \%$. Exploring oxygen needs in those with severe $\mathrm{PH}$ produced similar results. Combining the presence of systemic hypertension with the need for supplemental oxygen did not appear to improve screening efforts based on clinical criteria. For any $\mathrm{PH}$, the sensitivity and specificity of the combination of systemic hypertension and need for supplemental oxygen were 23.5 and $90.5 \%$, respectively. Although the sensitivity is increased with this approach, the accuracy drops to $41.0 \%$. Underscoring the potential limited role for clinical criteria, in severe $\mathrm{PH}$, use of both systemic hypertension and supplemental oxygen results in the misclassification of nearly one in three patients (e.g. overall accuracy 63.9\%).

\section{DISCUSSION}

This retrospective analysis of a large cohort of patients with advanced sarcoidosis demonstrates that both $\mathrm{PH}$ and severe elevations in Ppa are common in this setting. Nonetheless, simple clinical criteria have limited power as screening tests to identify those persons with sarcoidosis who concomitantly have $\mathrm{PH}$.

The mechanisms for $\mathrm{PH}$ in sarcoidosis remain unclear. As with other forms of interstitial lung disease, $P$ pa may rise as a result of progressive fibrosis and destruction of alveolar capillary membrane units [11]. Additionally, the present findings, regarding the incidence of $\mathrm{PH}$, are consistent with the observations by GLUSKOWSKI et al. [12] that $50 \%$ of those with stage III sarcoidosis have increased $P$ pa.

However, among those with moderate elevations of $P$ pa, the authors' failure to note a correlation between certain measures lung function and the results of invasive haemodynamic monitoring imply that alternative mechanisms are at play. In fact, in severe $\mathrm{PH}$, an inverse relationship was found between FVC and Ppa. A systemic vasculitis, which affects the pulmonary vasculature, can complicate sarcoidosis. FERNANDES et al. [13] have described this phenomenon in a detailed review of published reports of vasculitis in sarcoidosis. Based on arteriography, patients had evidence of both large and small vessel vasculitis. Many of these subjects, however, were children, whereas the current analysis was restricted to adults. Vasculitis is also exceedingly rare in sarcoidosis, with $<20$ such cases described in the English literature [13]. A more likely factor, which may contribute to the development of $\mathrm{PH}$ in sarcoidosis, is the heightened vasoresponsiveness of the pulmonary vasculature in this disease. In a prospective study of eight patients with sarcoidosis and $\mathrm{PH}$, PRESTON et al. [14] noted significant drops in Ppa in response to inhaled nitric oxide (iNO). Moreover, despite moderately preserved lung function in their cohort (mean FVC 50\% pred), the mean $P$ pa measured $55 \mathrm{mmHg}$, indicating a discordance between static measures of lung function and $\mathrm{PH}$. The current authors' observations confirm the limited relationship between lung function and $\mathrm{PH}$ in sarcoidosis.

Cardiac factors could also potentially explain $\mathrm{PH}$ in sarcoidosis. First, patients with sarcoidosis are at risk of direct myocardial involvement. Due to their lung disease, these individuals could additionally develop right-sided heart failure as a consequence of left ventricular systolic dysfunction. In multivariate analysis, however, the cardiac index was not an independent predictor of $\mathrm{PH}$ and implies that cardiac sarcoidosis was not a significant contributor to $\mathrm{PH}$. Furthermore, although the cardiac index was statistically lower in univariate analysis in patients with $\mathrm{PH}$, the mean $0.13 \mathrm{~L} \cdot \mathrm{min}^{-1} \cdot \mathrm{m}^{-2}$ difference may not necessarily be clinically insignificant. Secondly, and alternatively, the association between $P$ pcw and $\mathrm{PH}$ indicates that subtle impairment in cardiac performance may nonetheless exist. This relationship could possibly reflect left ventricular diastolic dysfunction and impaired relaxation. The nexus between $\mathrm{PH}$ and systemic hypertension observed in patients with severe $\mathrm{PH}$ supports this hypothesis regarding the significance of diastolic dysfunction. These observations also are consistent with the conclusions of SCHARF et al. [15]. In a detailed analysis of pulmonary haemodynamics in 120 subjects with advanced emphysema, these investigators demonstrated that $\mathrm{PH}$ was common and not associated with right or left systolic performance. However, they did note that diastolic dysfunction contributed to $\mathrm{PH}$ [14]. As with many analyses of pulmonary haemodynamics, in patients with sarcoidosis being evaluated for lung transplantation, the RHCs were performed at rest. Invasive testing carried out with exertion could reveal impairments and limitations in cardiac performance not evident at rest. Hence, the importance of systolic cardiac function in the evolution of $\mathrm{PH}$ in sarcoidosis cannot be entirely discounted.

In more severe $\mathrm{PH}$, cardiac systolic function may be more directly involved in the evolution in $\mathrm{PH}$. $\mathrm{PH}$ could simply reflect impaired forward flow and arise as a result of poor cardiac performance. If this were the case, it may be predicted that the Ppcw would, in turn, be elevated. However, despite the $P$ pcw being higher in univariate analysis among those with severe $\mathrm{PH}$, the $\mathrm{Ppcw}$ was not independently associated with the presence of severe $\mathrm{PH}$. PH could theoretically compromise cardiac output through altering ventricular shape. This phenomenon, ventricular interdependence, occurs when dysfunction of one ventricle affects the function of the other through decreasing diastolic filling. SCHARF et al. [15] did not observe ventricular interdependence in their analysis of patients with emphysema. Unfortunately, because of the lack of data regarding measures of ventricular performance (e.g. right and left ventricular end-diastolic indices), the present authors could not assess the degree to which ventricular interdependence is an issue in advanced sarcoidosis. Irrespectively, cardiac disease does not appear to fully account for the $\mathrm{PH}$ noted, as other variables are also independently correlated with the presence of $\mathrm{PH}$, and the degree of elevation in $P$ pa was out of proportion to the mild increase in Ppcw. Finally, the lower cardiac index in the $\mathrm{PH}$ group, while statistically significant, may be more likely to represent an effect of the patients' $\mathrm{PH}$ rather than being causative, akin to what is seen in patients with idiopathic pulmonary arterial 
hypertension. Without data from serial RHCs, differentiating cause from effect is difficult.

Hypoxaemic vasoconstriction might also contribute aetiologically to $\mathrm{PH}$ in sarcoidosis. Alveolar hypoxia, irrespective of the cause, can result in vasoconstriction and vascular remodelling. Clinically, however, persons with superimposed $\mathrm{PH}$ are more likely to require supplemental oxygen. Hence, the increased use of supplemental oxygen among patients with $\mathrm{PH}$ does not necessarily imply causation. In other words, it may simply represent an epiphenomenon and be a marker for this process.

The current authors' findings did detect a connection between functional status and $\mathrm{PH}$ in univariate analysis. This is not surprising given that $\mathrm{PH}(\mathrm{Ppa} \geqslant 40 \mathrm{mmHg})$ is known to be a debilitating condition irrespective of its aetiology. However, no prior studies have described the relationship between functional status and $\mathrm{PH}$ in sarcoidosis. The fact that nearly $70 \%$ of those with $P$ pa $\geqslant 40 \mathrm{mmHg}$ required some or total assistance with functional activities underscores the burden of $\mathrm{PH}$ in sarcoidosis. Therefore, clinicians who care for patients with advanced sarcoidosis must take steps to identify and treat this complication. For example, diagnosing $\mathrm{PH}$ when it is relatively mild could prompt physicians to begin an evaluation for occult oxygen desaturation and to consider more aggressive use of supplemental oxygen. Additionally, newer therapeutic options are available. PRESTON et al. [14] treated patients with long-term iNO and epoprostentol with favourable results. Similarly, endothelin receptor blockers have shown promise in the management of secondary PH [16]. In a trial of sildenafil, a phosphodiesterase inhibitor, in lung fibrosis and $\mathrm{PH}$, this agent led to preferential pulmonary vasodilation and increased the $\mathrm{Pa}_{\mathrm{a}} \mathrm{O}_{2}$ relative to epoprostenol [17].

The role for standard sarcoidosis therapies for $\mathrm{PH}$ in this disease is unclear. Some have reported success with corticosteroids, while others have not noted improvements in pulmonary haemodynamics with these agents [12, 14]. In the present analysis, despite more than half of patients receiving corticosteroids, $\mathrm{PH}$ was common. Additionally, there was no evident difference in the incidence of $\mathrm{PH}$ as a function of treatment with corticosteroids. This suggests that the role for corticosteroids in sarcoidosis-associated $\mathrm{PH}$ is likely to be limited. More importantly, given the impact of $\mathrm{PH}$ on both morbidity and mortality in sarcoidosis, coupled with enhanced treatment alternatives, it is essential that physicians consider evaluating their patients with sarcoidosis for this problem.

Optimally, any strategy to identify those with sarcoidosis and $\mathrm{PH}$ would begin by determining who is at a high risk for this condition. These individuals would then undergo further testing. Focusing on the high-risk population would improve the yield of more invasive interventions, while minimising the potential costs associated with more extensive evaluation. In short, the ideal approach would rely on screening tools with high accuracy. Unfortunately though, it does not appear that simple clinical criteria adequately segregate those with $\mathrm{PH}$ from those with normal Ppa. Relying on oxygen use, the only variable independently associated with $\mathrm{PH}$, leads to the misclassification of nearly one in three patients. In light of the prognostic implications of $\mathrm{PH}$ in sarcoidosis, such an error rate is unacceptable. Hence, simple clinical criteria, such as pulmonary function testing and performance status, cannot be used as a guide in determining whom requires further evaluation. Part of this arises because $\mathrm{PH}$ is so prevalent in the population of patients with sarcoidosis listed for OLT. At a minimum, therefore, the present authors recommend RHC for all patients being considered for OLT for sarcoidosis. Is there a role for ECG in other individuals suspected of having PH? ECG generally represents a reliable and accurate noninvasive tool for gauging $P$ pa. However, in advanced lung disease this may not be the case. In a prospective study of 374 people, ARCASOY et al. [18] reported that ECG allowed estimation of $P$ pa in only $44 \%$ of subjects. Among those with interstitial lung disease, the positive and negative predictive values of ECG for the diagnosis of $\mathrm{PH}$ equalled only 60 and 44\%, respectively [18]. Other potential markers for $\mathrm{PH}$ in this population, such as presence and extent of desaturation during a 6-min walk test and serum markers of heart failure (e.g. brain naturetic peptide), could represent less invasive screening options. As such, they merit formal study in prospective analyses of $\mathrm{PH}$ in interstitial lung disease.

The current study has several limitations. The retrospective design exposes the study to bias. However, the UNOS registry represents data collected contemporaneously at time of listing. Due to this, recall bias and coding bias are unlikely to be a concern. The current authors also lacked information on certain variables that could potentially have been of interest, such as the DL,CO. Similarly, the UNOS registry was not designed to investigate $\mathrm{PH}$. Therefore, some information, such as the presence of desaturation with exercise, the exact distance walked during a 6-min walk test, the actual amount and duration of corticosteroids used, and specific information on ventricular performance potentially obtainable from RHC, were not collected and, thus, not available for inclusion in the current analysis. Furthermore, the current authors studied only patients actually listed for OLT and lacked complete RHC information on all listed subjects. As such, there may be referral bias present, in that there was no data on subjects who were not referred for OLT. For example, some of the patients with the most severe impairments in lung function might have died prior to listing or been deemed too ill for transplantation. This fact may explain why the present authors failed to note a relationship between certain variables and $\mathrm{PH}$, and simultaneously limits the generalisability of the present conclusions. Despite these considerations, the current study represents the largest experience with RHC in sarcoidosis. All earlier studies on this topic are far smaller and consist of $<25$ patients [11-13, 15, 19-21]. These other reports also originate from single centres, while the current study encompasses multiple sites throughout the USA. Due to this increase in sample size, this study was able to explore issues such as functional and employment status, which have not previously been explored as they relate to $\mathrm{PH}$ in sarcoidosis.

In conclusion, pulmonary hypertension is common in patients with advanced sarcoidosis. Simple clinical criteria do not successfully function as a screening test for pulmonary hypertension in this disease. Based on present observations, the authors can conclude that pulmonary hypertension in sarcoidosis is multifactorial in origin and does not solely arise because of progressive lung fibrosis. 


\section{REFERENCES}

1 Thomas KW, Hunninghake GW. Sarcoidosis. JAMA 2003; 289: 3300-3303.

2 Statement on sarcoidosis. Joint Statement of the American Thoracic Society (ATS), the European Respiratory Society (ERS) and the World Association of Sarcoidosis and Other Granulomatous Disorders (WASOG) adopted by the ATS Board of Directors and by the ERS Executive Committee, February 1999. Am J Respir Crit Care Med 1999; 160: 736-755.

3 Baughman RP, Lower EE. A clinical approach to the use of methotrexate for sarcoidosis. Thorax 1999; 54: 742-746.

4 Baughman RP, Lower EE. Infliximab for refractory sarcoidosis. Sarcoidosis Vasc Diffuse Lung Dis 2001; 18: 70-74.

5 Meyerle JH, Shorr A. The use of infliximab in cutaneous sarcoidosis. J Drugs Dermatol 2003; 2: 413-414.

6 Maurer JR, Frost AE, Estenne M, Higenbottam T, Glanville AR. International guidelines for the selection of lung transplant candidates. The International Society for Heart and Lung Transplantation, the American Thoracic Society, the American Society of Transplant Physicians, the European Respiratory Society. Transplantation 1998; 66: 951-956.

7 Arcasoy SM, Christie JD, Pochettino A, et al. Characteristics and outcomes of patients with sarcoidosis listed for lung transplantation. Chest 2000; 120: 873-880.

8 Shorr AF, Davies DB, Nathan SD. Predicting mortality in patients with sarcoidosis awaiting lung transplantation. Chest 2003; 124: 922-928.

9 United Network for Organ Sharing. Proposals Archive. www.unos.org/policiesandbylaws/publiccomment/ proposalsarchive.asp. Date last updated: January 152004. Date last accessed: November 152003.

10 Shorr AF, Davies DB, Nathan SD. Outcomes for patients with sarcoidosis awaiting lung transplantation. Chest 2002; 122: $233-238$.
11 Gluskowski J, Hawrylkiewicz I, Zych D, Wojtczak A, Zielinski J. Pulmonary haemodynamics at rest and during exercise in patients with sarcoidosis. Respiration 1984; 46: 26-32.

12 Gluskowski J, Hawrylkiewicz I, Zych D, Zielinski J. Effects of corticosteroid treatment on pulmonary haemodynamics in patients with sarcoidosis. Eur Respir J 1990; 3: 403-407.

13 Fernandes SR, Singsen BH, Hoffman GS. Sarcoidosis and systemic vasculitis. Semin Arthritis Rheum 2000; 30: 33-46.

14 Preston IR, Klinger JR, Landzberg MJ, Houtchens J, Nelson D, Hill NS. Vasoresponsiveness of sarcoidosisassociated pulmonary hypertension. Chest 2001; 120: 866-872.

15 Scharf SM, Iqbal M, Keller C, et al. Hemodynamic characterization of patients with severe emphysema. Am J Respir Crit Care Med 2002; 166: 314-322.

16 Rubin LJ, Badesch DB, Barst RJ, et al. Bosentan therapy for pulmonary arterial hypertension. N Engl J Med 2002; 346: 896-903.

17 Ghofrani HA, Wiedemann R, Rose F, et al. Sildenafil for treatment of lung fibrosis and pulmonary hypertension: a randomised controlled trial. Lancet 2002; 360: 895-900.

18 Arcasoy SM, Christie JD, Ferrari VA, et al. Echocardiographic assessment of pulmonary hypertension in patients with advanced lung disease. Am J Respir Crit Care Med 2003; 167: 735-740.

19 Bjortuft O, Simonsen S, Geiran OR, Fjeld JG, Skovlund E, Boe J. Pulmonary haemodynamics after single-lung transplantation for end-stage pulmonary parenchymal disease. Eur Respir J 1996; 9: 2007-2011.

20 Rodman DM, Lindenfeld J. Successful treatment of sarcoidosis-associated pulmonary hypertension with corticosteroids. Chest 1990; 97: 500-502.

21 Jones K, Higenbottam T, Wallwork J. Pulmonary vasodilation with prostacyclin in primary and secondary pulmonary hypertension. Chest 1989; 96: 784-789. 